\title{
On The Mathematical Analysis of Black-Hole Information Loss Paradox
}

\author{
V. Vishal*, B. Siddharth, C. Venkatachalam \\ Sri Venkateswara College of Engineering, Pennalur, Irungattukottai - 602117 \\ Sriperumbudur Taluk, Tamilnadu, India \\ *E-mail address: vishal.145@hotmail.com
}

\begin{abstract}
A Black-hole is an astronomical entity which possesses infinite density at its gravitational singularity or singular point. The capacity of a black-hole to completely rip-off an entire solar system without leaving any evidence is to be noted. A debate has been going on over the past few decades regarding the information storage in black-holes. The discovery of Hawking radiation, which predicts complete evaporation of mass violates unitarity ie. Conservation of probability and energy fails. Recent discoveries suggest that regular remnant of black-hole survives evaporation, as a result information of the object devoured can be contained. These remnants are grouped into embedded submanifolds. These manifolds are the result of a five-dimensional constant curvature bulk in space-time. Five-dimensional gravity can be recovered from brane-world resulting from equations of bulk geometry. Gravity can be explained by space-time theory and also quantum theory in the form of Gravitons. On observing the manifold, the gravitons show deformations in dimensions, rather than being constant. The perturbations in geometry can be related to embedding functions which should remain differentiable and regular. Regularity is related to the inverse functions theorem. Manifold observations followed by a mathematical approach can possibly retain information about objects devoured by the black-hole.
\end{abstract}

Keywords: Black hole; Information paradox; AdS/CFT Correspondence; Quantum field theory

\section{INTRODUCTION}

A black hole is a region of space-time, which possesses an infinite gravitational intensity, so that even light photons do not escape [1]. It is formed as a result of gravitational collapse due to its own mass. The black hole is characterized by the point of no return which is popularly known as the event horizon. Any object approaching the black hole remains frozen in time as the body will take infite time to fall into the black hole. But, Quantum mechanics predicts that black holes emit radiation like a black body with a finite temperature. The temperature accompanying the radiation is small in magnitude, as the temperature is inversely proportional to its mass. This will make the astronomical observation of black-hole impossible due to the low temperature. 


\section{INFORMATION LOSS PHENOMENON}

As a black hole has only a few internal parameters most of the information about the matter that went into forming the black hole is lost. It does not matter if it is formed from television sets or chairs; in the end the black hole only remembers the total mass, charge, and angular momentum. As long as black holes were thought to persist forever this information loss is not that problematic, as the information can be thought of as existing inside the black hole, inaccessible from the outside. However, black holes slowly evaporate by emitting Hawking radiation [2]. However, the radiation does not give a detailed picture of the object lost. Fig. 1 shows the penrose diagram of a black hole along with the Hawking radiation.

There could be no solution this problem from the field of relativistic physics. But, quantum mechanics was found to be an effective tool to tackle this problem. In quantum mechanics, loss of information corresponds to the violation of vital property called unitarity, which has to do with the conservation of probability. It has been argued that loss of unitarity would also imply violation of conservation of energy [3]. But there are instances in which unitarity follows the laws of conservation. There are various approaches to this problem and no approach is an unmixed blessing. This attempt is only to understand the various concepts involved in Information Paradox and trying to establish a mathematical relation to this problem so that we reach the a milestone in relating information loss paradox. Fig. 1 shows the Penrose diagram, whcih shows the evaporation of a black hole due to Hawking Radiation and also the indiacation of the outgoing radiation and the incomig particle [4].

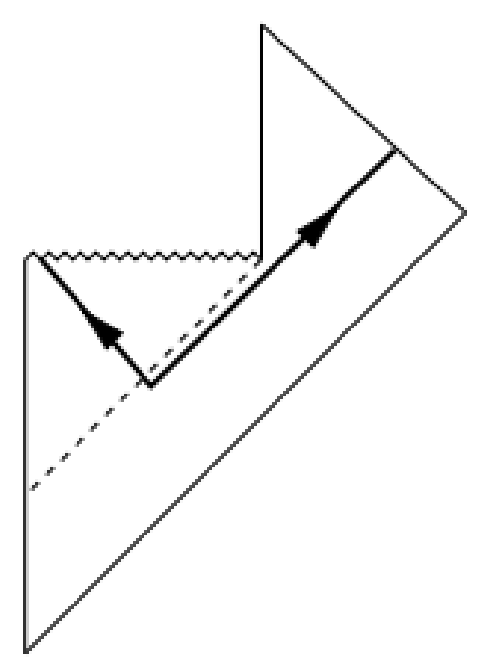

Fig. 1. Representation of a Schwarzschild black hole by the Penrose diagram.

\section{INFORMATION RETRIEVAL}

Information is generally encoded in a wave function. And the wave function can be extended to retrieve the information. Arguments were put forth on general field theory and the genral theory of relativity regarding the failure in conservation of information. However, the 
Holographic principle which was proposed by 't Hooft, strengthened by Susskind's string theory interpretation, gave an explanation to the information loss. Over the past few years, a few notable solutions have been framed to tackle this problem.

\section{1. Information stored in a parallel universe}

The advantage is that unitarity seemed to be conserved in this solution. But the solution was not backed by fundamental theories which predict the existence of a parallel universe [5].

\section{2. Information is encoded in the same space but a different time}

This solution violates our understanding about the fact that nature evolves with time [6]. However, the solution does not violate the conservation of energy.

\section{3. Information stored in remnants}

This solution does indicate that information can be retrieved in the same space-time. But the identification of remnants is difficult since they are small and indistinguishable. This particular idea has a specific advantage over the other methodologies. These remnants would have an infinite number of energy states. The unification of quantum mechanics and the string theory produces a dual nature of matter which eases examination of the remnants [3].

\section{DISCUSSION AND ANALYSIS}

\section{1. Unification Theory}

A tool which unifies gravity and string theory defined on one space, and a quantum field theory without gravity defined on the conformal boundary of the same space, can be used to analyze the problem [7]. The dimension of the boundary is lower than the dimension of the space. This duality is the AdS/CFT correspondence which relates anti de sitter spacetime theory and conformal field theory [8]. The Ads space includes closed manifolds like sphere or any non-commutative space. For example, Ads5 a 5-dimensional space shares duality with a 4-dimensional CFT, arising from the brane theory and the gauge theory. This helps in realizing the holographic principle. The remnants on a black hole which survive evaporation due to the Hawking radiation can be analyzed using this technique.

The 5-dimensional black hole forms the metric background. They have mass-less fields residing on it from CFT. From the establishments of IIB string theory, there is a relationship between particle theory and wave theory. As a result, the brane fields can be related to gravitons by the correspondence. The correspondence establishes a dependence of a CFT bound state of any size to a bulk-particle such as a graviton. The gravitons from the remnant are considered to show deformation. The deformation is predicted by the field theory. The deformations are not uniform and hence perturbation theory applied to their geometry can be used to arrive at an exact solution. They require embedding function which should remain regular and differential. To determine whether the embedding function is regular or not, the inverse functions theorem is applied. The deformations can be encoded to formulate a mathematical series. The series will provide solutions at different intervals.

\section{2. Mathematical Observation}

Topological Observation of a remnant which survives observation is made. The remnant possess a finite diameter determined by the fundamental energy scale. On considering the 
remnant as regular manifolds embedded in the bulk, information stored can be retrieved by the use of the local inverse of the embedding map. However, the manifold does not remain uniform throughout.

Let $\mathrm{M}$ and $\mathrm{N}$ be smooth manifolds and $\mathrm{f}: \mathrm{M} \rightarrow \mathrm{N}$ be a smooth map. The image of embedding results in a sub-manifold. For a point $\mathrm{x} € \mathrm{M}$, there is a neighborhood, $\mathrm{x} € \mathrm{U}$ which is a subset of $M$, ie. $f: U \rightarrow N$.

As the manifolds in the observed remnants are non-uniform, a distortion quotient $\mathrm{c}$ is involved. Eq. 1 shows the inequality relation involving the distortion quotient.

$$
d x f(x, y) \leq d y(f(x), f(y)) \leq c d x f(x, y)
$$

The embedded function is differentiable and regular. Regularity helps us in applying inverse functions theorem. Consider the embedded bulk. CFT and AdS5 relationship can be established by multiplying stress-energy operator to the field size, to extend a conformal mass. The stress-energy operator reduces the field size to negative curvature constant, which is related to the AdS5. A scalar operator with conformal weight $\Delta$ will be dual to a scalar bulk field with a bulk mass of $\mathrm{K} V[\Delta(\Delta-4)]$, as the field has one dimension lesser according the correspondence employed. The conformal weight of the stress-energy operator, $\Delta=4$.

Thus the bulk mass becomes zero which explains why gravitons have zero mass. From the correspondence, it is established that the CFT bound area of size $r$ is approximately same as a localized point $\mathrm{z}=\mathrm{r}$ on the bulk. The embedded bulk may contain numerous localized points.

These points can be grouped into a function $\mathrm{g}$.

$$
\mathrm{g}(\mathrm{z})=\{\text { localized points. }\}
$$

And we know that $\mathrm{z} €(\mathrm{x}, \mathrm{y})$ where $\mathrm{x}$ and $\mathrm{y}$ form the co-ordinates of the localize point.

This implies,

$$
\mathrm{f}(\mathrm{r})=\mathrm{g}(\mathrm{z})=\mathrm{g}(\mathrm{x}, \mathrm{y})
$$

where $\mathrm{f}$ is a function housing the various CFT bound area of different sizes.

The usage of inverse functions theorem enables us to obtain $(\mathrm{x}, \mathrm{y})$

$$
(x, y)=g^{-1}(f(r))
$$

and the general solution is denoted by Ao. However this solution is found to be approximate. The co-ordinate values are obtained for different localized points in the same iterated manner. A1, A2,... An are higher order solutions. From the theory of perturbations, the general solution can be expressed as a series which is given by,

$$
A=A o+\left(E^{1} \cdot A 1\right)+\left(E^{2} \cdot A 2\right)+\ldots \ldots \cdot
$$

where $\mathrm{E}$ is a small variation from the inital computation.

As the value of $\mathrm{E}$ is small, the values of higher solutions are smaller. 
A solution is almost accurate and is given by,

$$
\mathrm{A}=\mathrm{Ao}+\mathrm{E}(\mathrm{A} 1)
$$

\section{CONCLUSION}

Hence, we have combined various concepts such as that of the information stored in remnants, relating the information loss to the remnants, unification of quantum mechanics and string theory, brane theory, concept of gravitons and perturbation theory in getting a solution to Information loss in black holes. We also arrived at a series with terms and we have considered only the first two terms to get the nearly exact solution.

\section{References}

[1] Wald Robert M., General Relativity, University of Chicago Press, (1984) 299-300

[2] Hawking S. W, Nature 248 (2008) 30-31.

[3] Giddings S. B, "The black hole information paradox", Particles, Strings and Cosmology, Johns Hopkins Workshop on Current Problems in Particle Theory 19 and the PASCOS Interdisciplinary Symposium 5, (1995). arXiv:hep-th/9508151.

[4] Nikolić Hrvoje, Physics Letters B 678.2 (2009) 218-221.

[5] Preskill John, "Do Black Holes Destroy Information?". International Symposium on Black Holes, Membranes, Wormholes, and Superstrings (1992). arXiv:hep-th/9209058.

[6] Hartle James B., "Generalized Quantum Theory in Evaporating Black Hole Spacetimes". Black Holes and Relativistic Stars: 195 (1998). arXiv:gr-qc/9705022.

[7] Mukul Chandra Das, Rampada Misra, International Letters of Chemistry, Physics and Astronomy 9(1) (2013) 13-16.

[8] Maldacena Juan, "The Illusion of Gravity". Scientific American (2005). 